\title{
Erratum: Physics from solar neutrinos in dark matter direct detection experiments
}

\author{
David G. Cerdeño, ${ }^{a}$ Malcolm Fairbairn, ${ }^{b}$ Thomas Jubb, ${ }^{a}$ Pedro A.N. Machado, ${ }^{c, d}$ \\ Aaron C. Vincent ${ }^{a}$ and Céline Bœhm ${ }^{a, e}$ \\ ${ }^{a}$ Institute for Particle Physics Phenomenology (IPPP), Durham University, \\ Durham DH1 3LE, U.K. \\ ${ }^{b}$ Physics, King's College London, \\ Strand, London, WC2R 2LS, U.K. \\ ${ }^{c}$ Departamento de Fúsica Teórica, Universidad Autónoma de Madrid, \\ Cantoblanco E-28049 Madrid, Spain \\ ${ }^{d}$ Instituto de Física Teórica UAM/CSIC, \\ Calle Nicolás Cabrera 13-15, Cantoblanco E-28049 Madrid, Spain \\ ${ }^{e}$ LAPTH, Université de Savoie, CNRS, \\ BP 110, 74941 Annecy-Le-Vieux, France \\ E-mail: davidg.cerdeno@gmail.com, malcolm.fairbairn@kcl.ac.uk, \\ thomas.jubb@durham.ac.uk, pedro.machado@uam.es, \\ aaron.vincent@durham.ac.uk, c.m.boehm@durham.ac.uk
}

ERRATUM TO: JHEP05(2016)118

ABSTRACT: We correct 1) missing factors of 2 in cross section expressions in table 4 of the published article; and 2) the LUX bound in figure 6 on the B-L coupling from 2015 data, which displayed erroneously strong constraints due to a computer error. Conclusions remain unchanged.

ARXIV EPRINT: 1604.01025 


\begin{tabular}{|c|c|c|c|}
\hline Mediator & $\mathcal{L}$ & $d \sigma_{e} / d E_{R}-d \sigma_{e}^{\mathrm{SM}} / d E_{R}$ & $d \sigma_{N} / d E_{R}-d \sigma_{N}^{\mathrm{SM}} / d E_{R}$ \\
\hline Scalar & $\begin{array}{l}\left(g_{\nu, \phi} \phi \bar{\nu}_{R} \nu_{L}+h . c .\right) \\
+\phi \bar{\ell} g_{\ell, s} \ell+\phi \bar{q} g_{q, s} q\end{array}$ & $\frac{g_{\nu, \phi}^{2} g_{e_{, s}}^{2} E_{R} m_{e}^{2}}{4 \pi E_{\nu}^{2}\left(2 E_{R} m_{e}+m_{\phi}^{2}\right)^{2}}$ & $\frac{Q_{s}^{\prime 2} m_{N}^{2} E_{R}}{4 \pi E_{\nu}^{2}\left(2 E_{R} m_{N}+m_{\phi}^{2}\right)^{2}}$ \\
\hline Pseudoscalar & $\begin{array}{l}\left(g_{\nu, \phi} \phi \bar{\nu}_{R} \nu_{L}+h . c .\right) \\
-i \gamma^{5} \phi \bar{\ell} g_{\ell, p} \ell-i \gamma^{5} \phi \bar{q} g_{q, p} q\end{array}$ & $\frac{g_{\nu, \phi}^{2} g_{e, p}^{2} E_{R}^{2} m_{e}}{8 \pi E_{\nu}^{2}\left(2 E_{R} m_{e}+m_{\phi}^{2}\right)^{2}}$ & 0 \\
\hline Vector & $\begin{array}{l}g_{\nu, Z^{\prime}} Z_{\mu}^{\prime} \bar{\nu}_{L} \gamma^{\mu} \nu_{L} \\
+\quad Z_{\mu}^{\prime} \bar{\ell} \gamma^{\mu} g_{\ell, v} \ell \\
Z_{\mu}^{\prime} \bar{q} \gamma^{\mu} g_{q, v} q\end{array}+$ & $\begin{array}{l}\frac{\sqrt{2} G_{F} m_{e} g_{v} g_{\nu, Z^{\prime}} g_{e, v}}{\pi\left(2 E_{R} m_{e}+m_{Z^{\prime}}^{2}\right)} \\
+\frac{m_{e} g_{\nu, Z^{\prime}}^{2} g_{e, v}^{2}}{2 \pi\left(2 E_{R} m_{e}+m_{Z^{\prime}}^{2}\right)^{2}}\end{array}$ & $\begin{array}{l}-\frac{G_{F} m_{N} Q_{v} Q_{v}^{\prime}\left(2 E_{\nu}^{2}-E_{R} m_{N}\right)}{2 \sqrt{2} \pi E_{\nu}^{2}\left(2 E_{R} m_{N}+m_{Z^{\prime}}^{2}\right)} \\
+\frac{Q_{v}^{\prime 2} m_{N}\left(2 E_{\nu}^{2}-E_{R} m_{N}\right)}{4 \pi E_{\nu}^{2}\left(2 E_{R} m_{N}+m_{Z^{\prime}}^{2}\right)^{2}}\end{array}$ \\
\hline Axial Vector & $\begin{array}{l}g_{\nu, Z^{\prime}} Z_{\mu}^{\prime} \bar{\nu}_{L} \gamma^{\mu} \nu_{L} \\
-Z_{\mu}^{\prime} \bar{\ell} \gamma^{\mu} g_{\ell, a} \gamma^{5} \ell \\
-Z_{\mu}^{\prime} \bar{q} \gamma^{\mu} g_{q, a} \gamma^{5} q\end{array}$ & $\begin{array}{l}\frac{\sqrt{2} G_{F} m_{e} g_{a} g_{e, a} g_{\nu, Z^{\prime}}}{\pi\left(2 E_{R} m_{e}+m_{Z^{\prime}}^{2}\right)} \\
+\frac{m_{e} g_{\nu, Z^{\prime}}^{2} g_{e, a}^{2}}{2 \pi\left(2 E_{R} m_{e}+m_{Z^{\prime}}^{2}\right)^{2}}\end{array}$ & $\begin{array}{l}\frac{G_{F} m_{N} Q_{a} Q_{a}^{\prime}\left(2 E_{\nu}^{2}+m_{N} E_{R}\right)}{2 \sqrt{2} \pi E_{\nu}^{2}\left(2 E_{R} m_{N}+m_{Z^{\prime}}^{2}\right)} \\
-\frac{G_{F} m_{N} Q_{v} Q_{a}^{\prime} E_{\nu} E_{R}}{\sqrt{2} \pi E_{\nu}^{2}\left(2 E_{R} m_{N}+m_{Z^{\prime}}^{2}\right)} \\
+\frac{Q_{a}^{\prime 2} m_{N}\left(2 E_{\nu}^{2}+E_{R} m_{N}\right)}{4 \pi E_{\nu}^{2}\left(2 E_{R} m_{N}+m_{Z^{\prime}}^{2}\right)^{2}} \\
\end{array}$ \\
\hline
\end{tabular}

Table 4. New Lagrangian terms and differential cross sections with the nucleus $N$ and electron $e$ for the four types of new mediator we consider. Note the negative interference in the vector and axial case with the SM contribution. 


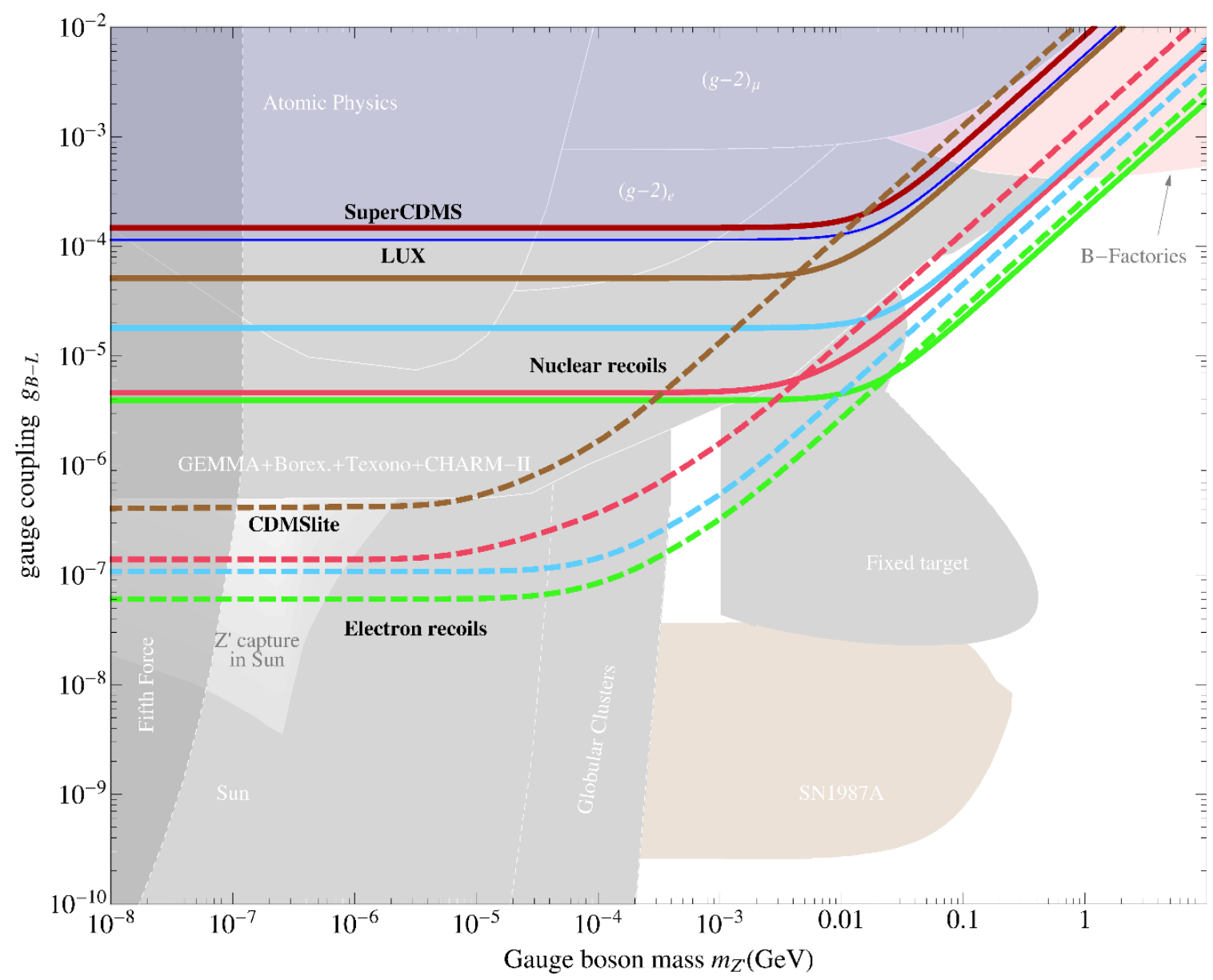

Figure 6. Projected 90\% CL constraints on the B-L model for nuclear recoils (solid lines) and electron recoils (dashed) in the optimistic scenarios for G2 germanium (red), G2 xenon (blue) and future xenon (green). We also show approximate bounds derived from the current SuperCDMS (red line), CDMSlite (brown solid and dashed lines), and LUX data (blue shaded region). Our bounds are overlaid on existing constraints. To translate these bounds to the other possible scenarios, one should keep in mind that some bounds (intermediate grey) only apply when the new mediator couples to leptons. The supernova bound (brown) only applies to couplings to baryons, while Bfactory bounds (pink) require both. The fifth force constraint (dark grey) applies in either case. The grey regions, the neutrino scattering bound and the pink pregion, and the supernova limits are respectively taken from refs. [1, 2], and [3].

\section{Acknowledgments}

We thank James Dent for pointing out the mistakes in table 4.

Open Access. This article is distributed under the terms of the Creative Commons Attribution License (CC-BY 4.0), which permits any use, distribution and reproduction in any medium, provided the original author(s) and source are credited. 


\section{References}

[1] R. Harnik, J. Kopp and P.A.N. Machado, Exploring $\nu$ signals in dark matter detectors, JCAP 07 (2012) 026 [arXiv: 1202.6073] [INSPIRE].

[2] S. Bilmis, I. Turan, T.M. Aliev, M. Deniz, L. Singh and H.T. Wong, Constraints on dark photon from neutrino-electron scattering experiments, Phys. Rev. D 92 (2015) 033009 [arXiv: 1502.07763] [INSPIRE].

[3] E. Rrapaj and S. Reddy, Nucleon-nucleon bremsstrahlung of dark gauge bosons and revised supernova constraints, arXiv:1511.09136 [INSPIRE]. 